\title{
Editorial
}

\section{Transformación digital: una agenda de oportunidades para la investigación y la práctica}

\section{OSCAR ANDRÉS GALINDO RODRÍGUEZ*}

En los últimos años, la transformación digital se ha convertido en un fenómeno importante en la investigación y en la práctica de diferentes campos y disciplinas. Por ello se entiende que su impacto abarca profundos cambios que tienen lugar en la sociedad y en las industrias, los cuales son generados por el uso de tecnologías digitales.

La mayoría de las tecnologías digitales relacionadas con la transformación digital se ajustan al acrónimo SMACIT; acrónimo que se refiere a las tecnologías de redes sociales, móviles, analítica, computación en la nube e Internet de las cosas -IoT-(Sebastian etal., 2017); las cuales, en el contexto de las organizaciones, son fuentes de disrupción en tres aspectos tal como lo afirman Vial (2019) y Verhoef et al. (2019):

(i) Alterando el comportamiento y las expectativas del consumidor: utilizando estas tecnologías, los consumidores se convierten en participantes activos en un diálogo que se lleva a cabo entre una organización y sus partes interesadas; los consumidores se han vuelto más conectados e informados; ya no se ven a sí mismos como cautivos de las organizaciones con las que realizan transacciones; permiten a los consumidores cocrear valor, diseñando y personalizando productos; sus expectativas con respecto a los productos o servicios están aumentando. Como resultado, anticipar en lugar de responder a los cambios en las expectativas de los clientes se ha convertido en un imperativo estratégico para las organizaciones.

(ii) Alterando el panorama competitivo: las tecnologías digitales facilitan la (re)combinación de productos y servicios existentes paragenerar nuevas formas de ofertas digitales; reducen las barreras de entrada; erosionan las fronteras entre industrias; permite que organizaciones digitales relativamente jóvenes tengan mayor protagonismo en el mercado frente a las organizaciones más conservadoras; facilitan que la competencia se vuelva más global; obstaculizan la sostenibilidad de ventajas competitivas.
COMO CITAR ESTE ARTÍCULO How to cite this article:

Galindo, O.A. (2020). Editorial. Transformación digital: una agenda de oportunidades para la investigación y la práctica. Revista Perspectiva Empresarial, 7(2), 3-6.

* Doctorando en Administración. Fundación Universitaria CEIPA, Sabaneta, Colombia. E-mail: oscar.galindo@ceipa.edu.co. ORCID: 0000-0003-3866-0461. Google Scholar: https://scholar.google.com/citations?hl=es\&user=RgmKusQAAAAJ. 
(iii) Aumentar la disponibilidad de datos: las tecnologías digitales también fomentan la generación de datos, lo que le permite a las organizaciones mediante su análisis y uso ofrecer servicios que respondan mejor a las necesidades de sus clientes; mejorar la velocidad en la toma de decisiones; realizar procesos de manera más eficiente en tiempo real.

Frente a este escenario, el cumplimiento de las estrategias y objetivos de las organizaciones se ven amenazados y socavados; lo anterior, implica una necesidad de cambios en los modelos de gestión y las arquitecturas organizacionales establecidas. Las tecnologías digitales traen un amplio espectro de oportunidades de negocio, junto con serios desafíos organizacionales, que se pueden abordar desde la transformación digital.

\section{Concepto de transformación digital}

Sin pretender agotar la discusión sobre el concepto de transformación digital y haciendo alusión a la investigación de Warner y Wäger (2019) que reveló que el término "transformación digital" es utilizado de manera inconsistente por los líderes dentro y en todos los contextos de la industria para describir diversas actividades de estrategia y organización; esto, "evidencia una desalineación general en el pensamiento estratégico, la planificación y la acción sobre este tema" (Warner and Wäger, 2019, p. 344).

De acuerdo con lo anterior, se presentan algunas definiciones que dan cuenta de su complejidad:

una definición reconocida es la de Fitzgerald et al. (2013): "el uso de nuevas tecnologías digitales (redes sociales, dispositivos móviles, análisis o dispositivos integrados) para permitir importantes mejoras comerciales, como mejorar la experiencia del cliente, racionalizar las operaciones o crear nuevos modelos de negocio" (p. 2).

Retomando a Warner y Wäger (2019), quienes definen la transformación digital como "un proceso continuo de renovación estratégica que utiliza los avances en las tecnologías digitales para desarrollar capacidades que actualizan o reemplazan el modelo comercial, el enfoque colaborativo y la cultura de una organización" (p. 344).

Por su parte, Vial (2019) expone conceptualmente la transformación digital como "un proceso que tiene como objetivo mejorar una entidad mediante la activación de cambios significativos en sus propiedades a través de combinaciones de tecnologías de información, informática, comunicación y conectividad" (p.118).

Por último, Verhoef et al. (2019) la describen como "un fenómeno de toda la empresa con amplias implicaciones organizativas en el que, sobre todo, el modelo de negocio principal de la empresa está sujeto a cambios a través del uso de tecnología digital" (p. 4).

Hastaahora Verhoefetal.(2019) puntualizan que la mayor atención del estudio de la transformación digital se ha dado dentro de disciplinas específicas tales como el marketing, la gestión estratégica, sistemas de información, innovación y gestión de operaciones. Pero diferentes autores plantean que se requiere una discusión multidisciplinaria sobre la transformación digital porque su naturaleza implica cambios en la estrategia, la organización, las tecnologías, el modelo de negocio, la cultura organizacional, según las definiciones anteriores.

En relación con esto, Singh y Hess (2017) sugieren que el término 'transformación' -en lugar de 'cambio' - enfatiza que la transformación digital de una organización va mucho más allá del pensamiento funcional y considera holísticamente la "integridad de las acciones" que deben tomarse para explotar las oportunidades o evitar las amenazas que se derivan de las tecnologías digitales.

\section{Implicaciones de la transformación digital en las organizaciones}

Las organizaciones más tradicionales han sido superadas por las nuevas organizaciones digitales de rápido crecimiento y han sufrido como resultado de esto. "Los directivos de muchas organizaciones han reconocido que la respuesta a los desafíos de 
la transformación digital determinará el éxito o el fracaso futuro de sus empresas" (Lichtenthaler, 2020 , p. 20). La variedad de cambios que implica la transformación digital expone a las organizaciones a enfrentarse a dificultades sustanciales, las cuales no solo son internas sino externas.

A nivel externo, por mencionar algunas dificultades, las organizaciones se enfrentan a una multitud de posibilidades tecnológicas; muchas de las cuales convergen; y esta convergencia complica aún más la planificación tecnológica estratégica. Por otro lado las empresas deben hacer frente a una variedad de transformaciones del mercado jalonadas por el comportamiento y las expectativas del consumidor y por la competencia cada vez más digital. Las organizaciones deben desarrollar respuestas estratégicas convincentes para poder responder de manera dinámica a los cambios del mercado y el entorno.

A nivel interno, los desafíos organizacionales también son sustanciales. La investigación de Vial (2019) demuestra que la tecnología en sí misma es solo una parte del complejo rompecabezas que deben resolver las organizaciones para que sigan siendo competitivas en un mundo digital. No se trata solo de la adquisición y uso de tecnología: la estrategia, la estructura organizacional, los procesos, el tipo de liderazgo y la cultura organizacional, son fundamentales para generar nuevos caminos para la creación de valor.

En ese contexto de transformación digital, los cambios en la organización también afectan de manera sustancial a las personas: los colaboradoreso empleados deben asumir roles que tradicionalmente estaban fuera de sus funciones; a medida que las tecnologías digitales permiten nuevas formas de hacer las cosas, preguntas sobre la necesidad de desarrollar las habilidades de los trabajadores existentes y las habilidades requeridas para los futuros trabajadores que formarán la fuerza laboral digital también son cada vez más relevantes. Lejos de eliminar la necesidad de que las organizaciones dependan del capital humano, la transformación digital requiere que los colaboradores o empleados dependan más de sus habilidades analíticas para resolver problemas organizacionales cada vez más complejos; de ahí que deben ser acompañados a través de esta transición (Singh and Hess, 2017). Esto implica otro desafío, la transformación del "lugar de trabajo digital" que se entiende como un fenómeno de las nuevas tecnologías que provocan cambios significativos en una variedad de aspectos relacionados con el trabajo: cambios en la forma en que los empleados realizan tareas y procesos; al igual que cambios en sus relaciones sociales, relaciones dentro de la organización y posteriormente a su experiencia laboral general. Como dice la literatura reciente, el futuro lugar de trabajo "se centra en cómo y qué trabajo se hace, no dónde y cuándo se hace" (Meske and Junglas, 2020, p. 1).

Finalmente la complejidad de la transformación digital posibilita una agenda amplia de oportunidades en investigaciones que aporten a su comprensión y práctica; los investigadores pueden hacer contribuciones desde diferentes disciplinas, perspectivas y lentes teóricos. Dada la naturaleza multidisciplinaria de la transformación digital y las interdependencias que existen en las organizaciones se requiere que los investigadores de diferentes campos trabajen juntos para no solo expandir el conocimiento sino para establecer activamente vínculos que permitan desarrollar una comprensión más integral de por qué, cómoy cuándo funciona. La investigación de transformación digital se puede desarrollar a nivel macro al observar los modelos de negocio y su impacto en la sociedad; a nivel medio, observando estructuras y procesos organizacionales; y a nivel micro, en el entorno laboral del individuo y en el conjunto de nuevas herramientas digitales que apoyan o dificultan la forma en que se realiza el trabajo.

La investigación multidisciplinaria $-\mathrm{y}$ en diferentes niveles - ayudará a los profesionales, consultores, gerentes y organizaciones a tomar decisiones estratégicas sólidas sobre cómo responder a las tecnologías digitales e implementar el cambio con la transformación digital.

\section{Referencias}

Fitzgerald, M. et al. (2013). Embracing Digital Technology: A New Strategic Imperative. MIT Sloan Management Review, 55(1), 1-13.

Lichtenthaler, U. (2020). Building Blocks of Successful Digital Transformation: Complementing Technology and Market Issues. International 
Journal of Innovation and Technology Management, 17(1), 2050004.

Meske, C. and Junglas, I. (2020). Investigating the elicitation of employees' support towards digital workplace transformation. Behaviour \& Information Technology, 39(4), 1-17.

Sebastian, I.M. et al. (2017). How big old companies navigate digital transformation. MIS Quarterly Executive, 16(3), 197-213.

Singh, A. and Hess, T. (2017). How chief digital officers promote the digital transformation of their companies. MIS Quarterly Executive, 16(1), 1-17.

Verhoef, P.C. et al. (2019). Digital transformation: A multidisciplinary reflection and research agenda. Journal of Business Research, 122, 889-901.

Vial, G. (2019). Understanding digital transformation: A review and a research agenda. Journal of Strategic Information Systems, 28(2), 118-144.

Warner, K.S.R. and Wäger, M. (2019). Building dynamic capabilities for digital transformation: An ongoing process of strategic renewal. Long Range Planning, 52(3), 326-349. 